\title{
FRA1 in macrophages promotes arthritis
}

A sophisticated transcriptional network controlled by several transcription factors enables macrophages to respond and adapt to their surrounding environment. One such transcription factor, Fos-related antigen 1 (FRA1), promotes a pro-inflammatory status in macrophages and impedes the resolution of arthritis in mice, according to results of a new study.

FRA1 has a known function in regulating cytokine expression in macrophages. "As macrophages have pro-inflammatory and destructive potential during rheumatoid arthritis (RA), we wondered if FRA1 could alter the responses of macrophages and the outcome of inflammation and bone and cartilage degeneration during arthritis," explains Nicole Hannemann, first author of the study.
The researchers investigated the effect of macrophage-specific deletion of FRA1 in the K/BxN serum-induced mouse model of arthritis. Compared with wild-type mice, the FRA1-deficient mice had less severe arthritis, including reduced immune cell infiltration and bone erosion.

In vitro analysis revealed that FRA1 inhibits the expression of the enzyme arginase 1 (ARG1). Indeed, the activity of ARG1 was increased in FRA1-deficient mice compared with wild-type mice. ARG1 catalyses the conversion of $\mathrm{L}$-arginine to L-ornithine and has known immunoregulatory functions.

Treatment of FRA1-deficient mice with an arginase inhibitor increased the severity of arthritis to the extent present in wild-type mice.

Given these findings, the authors investigated the effect of increasing

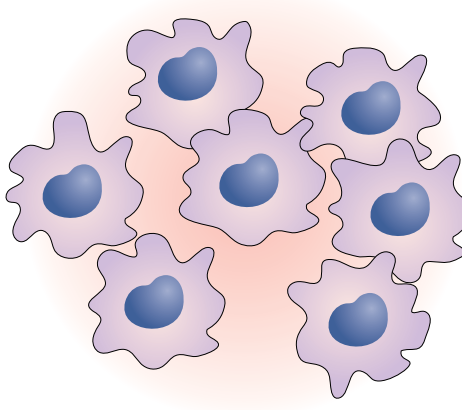

arginase activity through L-arginine supplementation either at arthritis onset or at the peak of inflammation. "We were excited to see that both approaches reduced inflammation and bone degeneration," says Hannemann.

"These results are promising and might provide therapeutic opportunities for altering macrophage responses from a pro-inflammatory to a pro-resolving state, which could induce the resolution of inflammation in RA but also in other inflammatory disease," concludes Hannemann.

\section{FRA 1-deficient mice had less severe arthritis

Jessica McHugh

ORIGINAL ARTICLE Hannemann, N. et al. Transcription factor Fra-1 targets arginase-1 to enhance macrophage-mediated inflammation in arthritis. J. Clin. Invest. https://doi.org/10.1172/ JCl96832 (2019)

\section{Choline uptake is vital for IL-1 $\beta$-driven inflammation}

Inhibition of choline uptake and phosphorylation prevents production of IL- $1 \beta$ and IL- 18 by activated macrophages, according to the results of a study published in Cell Metabolism.

Choline is an essential human nutrient; its uptake is mediated by choline transporters, including CTL1. Previous background work has shown that CTL1 expression is strongly induced during macrophage activation but the

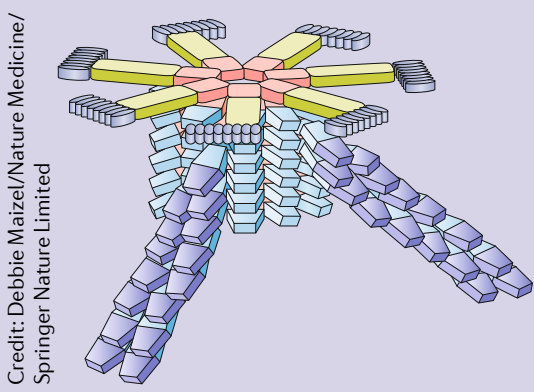

effect of choline uptake on macrophage biology was unknown.

Inhibition of choline uptake prevents

NLRP3 inflammasome activation and IL-1 $\beta$ and IL-18 production

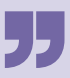

"Our experimental approach [was] based on standard CTL1 knockdown experiments that we complemented by the use of choline-free medium and choline kinase inhibitors," says Michael Karin, corresponding author. In a series of in vitro experiments, the researchers demonstrated that impaired choline uptake alters the mitochondrial lipid profile by reducing levels of mitochondrial phosphatidylcholine and increasing levels of mitochondrial sphingomyelin. Importantly, inhibiting choline uptake reduces synthesis of mitochondrial ATP and stimulates AMPK activation. AMPK stimulates mitophagy, leading to the removal of damaged mitochondria and the NLRP3 activating ligand ox-mtDNA (oxidized mitochondrial DNA). Thus, inhibition of choline uptake prevents NLRP3 inflammasome activation and IL-1 $\beta$ and IL-18 production.

In vivo experiments confirmed the preliminary findings. A choline kinase inhibitor attenuated inflammation in two acute experimental models of IL-1 $\beta$-dependent inflammation (lipopolysaccharide-induced septic shock and air-pouch gouty arthritis). Notably, treatment with a choline kinase inhibitor also ameliorated pathogenesis in a mouse model of Muckle-Wells syndrome (a cryopyrin-associated periodic syndrome that arises from NLRP3 gene mutations causing inflammasome activation).

"These results provide a direct demonstration that induction of mitophagy can be used to prevent and reverse inflammation in vivo," explains Karin. "We are planning to test the efficacy of choline kinase inhibitors in a number of IL-1 $\beta$ and IL-18-dependent diseases, especially lupus nephritis, osteoarthritis and Alzheimer disease."

Isobel Leake

ORIGINAL ARTICLE Sanchez-Lopez, E. et al. Choline uptake and metabolism modulate macrophage IL-1 $\beta$ and IL-18 production. Cell Metab. 29, 1-13 (2019) 